\title{
Co-annihilating Dark Matter: Effective Operator Analysis and Collider Phenomenology
}

\author{
Nicole F. Bell, ${ }^{1, *}$ Yi Cai ${ }^{1},{ }^{\dagger}$ and Anibal D. Medina ${ }^{1, \ddagger}$ \\ ${ }^{1}$ ARC Centre of Excellence for Particle Physics at the Terascale, \\ School of Physics, The University of Melbourne, Victoria 3010, Australia
}

\begin{abstract}
We study dark matter (DM) models in which there are two dark sector particles, $\chi_{1}$ and $\chi_{2}$, of near mass. In such models, co-annihilation of $\chi_{1}$ and $\chi_{2}$ may be the dominant process controlling the DM relic density during freezeout in the early universe. In this scenario, there is no significant contribution to direct and indirect detection signals, unless there exists an extreme degeneracy in the masses of the lightest dark sector particles. Therefore, relic density constraints and collider searches provide the most relevant information about these models. We consider Dirac fermion dark matter which couples to standard model (SM) particles via an effective operator. For the collider phenomenology, where an effective field theory may not be valid, we adopt a simple Z' model to provide an appropriate UV completion. We explore the interesting LHC signals that arise from the dark matter production process $p p \rightarrow \overline{\chi_{1}}+\chi_{2}+$ jet, followed by the decay $\chi_{2} \rightarrow \chi_{1}+S M$.
\end{abstract}

\section{INTRODUCTION}

Despite significant experimental effort to uncover the nature of dark matter via direct detection [1-9], collider searches [10-14], and indirect detection signals [15-23], it still remains elusive. The predictions for the signals in these experiments are, in general, quite model dependent. However, an effective field theory (EFT) formalism is a useful way to link results from these searches in a model independent framework [24-28].

In the EFT approach, we use a set of nonrenormalizable effective operators to parametrise the interaction of a pair of DM particles with standard model (SM) particles. The EFT operators are constrained only by Lorentz and gauge invariance, and would be obtained from a UV complete theory by integrating out the particle that mediates the interaction. For example, if we assume the dark matter is fermionic, the lowest order effective operators involving the DM, $\chi$, and SM fermions, $f$, are of the form

$$
\frac{1}{\Lambda_{\text {eff }}^{2}}\left(\bar{\chi} \Gamma_{1} \chi\right)\left(\bar{f} \Gamma_{2} f\right),
$$

where $\Gamma_{1}$ and $\Gamma_{2}$ are combinations of Dirac matrices. These operators are suppressed by two powers of an effective scale

$$
\Lambda_{\mathrm{eff}}=\frac{M}{\sqrt{g_{S M} g_{D M}}},
$$

where $M$ is the mass of the heavy mediator, which couples to the DM and SM particles with strength $g_{S M}$ and $g_{D M}$ respectively.

However, while the simple EFT approach is a useful starting point for describing DM interactions, it may

\footnotetext{
*n.bell@unimelb.edu.au

† yi.cai@unimelb.edu.au

$\ddagger$ amedina@physics.unimelb.edu.au
}

prove to be an inadequate description. For many choices of the effective operator, the parameter space is now quite constrained, particularly for low values of the dark matter mass. There is some tension between the lower limits on $\Lambda_{\text {eff }}$ obtained from the absence of signals in direct detection and colliders experiments, and the upper limits on $\Lambda_{\text {eff }}$ necessary to have sufficient annihilation in the early Universe such that the relic density does not lead to overclosure [29, 30]. Moreover, it is dangerous to blindly apply an EFT approach to collider studies [31]. Given that the effective scale $\Lambda_{\text {eff }}$ can be as light as several hundred $\mathrm{GeV}$, the momentum transfer in LHC interactions may easily exceed the mass of the particle which mediates the interaction.

An implicit assumption in the standard EFT description is a separation of scales which enables all the dark sector particles other than the DM candidate itself to be integrated out. This will break down if there are other dark sector particles with masses comparable to the DM. A well motivated example is the co-annihilation scenario, in which the dark sector contains two near-mass particles, $\chi_{1}$ and $\chi_{2}$, whose freezeout in the early universe is coupled $[32,33]$. The relic density is controlled by the (co-) annihilation processes, $\bar{\chi}_{i} \chi_{j} \rightarrow S M$, for $i, j=\{1,2\}$, rather than $\bar{\chi}_{1} \chi_{1}$ annihilation alone. With $m_{\chi_{2}} \gtrsim m_{\chi_{1}}$, the $\chi_{2}$ all decay to the stable DM candidate $\chi_{1}$, which forms the relic density in the universe today. However, because the relic density can be controlled by processes involving $\chi_{2}$, while the direct and indirect detection signals involve $\chi_{1}$ alone, the tensions between relic density and direct/indirect detection constraints are alleviated.

For the usual case of self-annihilating dark matter, collider constraints on $\Lambda_{\text {eff }}$ are obtained from monojet $[11,13,28,34,35]$, mono-photon $[12,35,36]$, or mono$\mathrm{W} / \mathrm{Z}$ [35, 37-40] searches for DM production. These signals are obtained when a single object, produced via initial state radiation (ISR) of a gluon, photon, or electroweak gauge boson, recoils against the missing transverse momentum attributed to dark matter.

In contrast, the co-annihilation model will have dis- 
tinct collider signals due the decay $\chi_{2} \rightarrow \chi_{1}+S M$. In principle, production of $\bar{\chi}_{i} \chi_{2}$ could be identified by the SM particles produced in $\chi_{2}$ decay, plus missing energy carried off by the $\chi$. However, if the mass difference $\Delta m_{\chi}=m_{\chi_{2}}-m_{\chi_{1}}$ is relatively low, $O(10 \mathrm{GeV})$, the SM particles will be soft and the missing $E_{T}$ low. While this signal could potentially be detected at a future lepton collider, it would be hidden in QCD backgrounds at a hadronic machine such as the LHC. We shall therefore be interested in the pair production of $\overline{\chi_{i}} \chi_{j}$ together with ISR, followed by $\chi_{2}$ decay. The ISR ensures a sufficiently large missing $E_{T}$ to allow the signal to be identified. This leads to distinct collider signatures:

(i) standard mono-jet plus $E_{T}$ signals result from $\bar{\chi}_{1} \chi_{1} j$ production, or from $\bar{\chi}_{1} \chi_{2} j$ and $\bar{\chi}_{2} \chi_{2} j$ where the $\chi_{2}$ decays invisibly (e.g. to neutrinos) or to quarks or leptons which are too soft to detect.

(ii) jet $+E_{T}+\bar{f} f$ results from $\bar{\chi}_{1} \chi_{2} j$ production.

(iii) jet $+E_{T}+\bar{f} f \overline{f^{\prime}} f^{\prime}$ results from $\bar{\chi}_{2} \chi_{2} j$ production.

These signals provide complementary information about the dark matter model. The mono-jet plus $E_{T}$ signal is of course not unique to the co-annihilation scenario. However, the observation of signal (ii) or (iii), together with (i), could be interpreted as evidence for co-annihilation. We shall study process (ii) in detail.

An outline of the paper is as follows: We define EFT and UV complete versions of our model in section II, compute relic density constraints in section III, and determine di-jet/di-lepton based coupling bounds in section IV. Finally, in section V, we explore co-annihilation collider signals at the LHC (process (ii) above).

\section{EFFECTIVE FIELD THEORY AND UV COMPLETION}

We generalize the standard EFT treatment to the case of co-annihilation by considering the following effective operators:

$$
\begin{aligned}
& \frac{1}{\Lambda_{11}^{2}}\left(\overline{\chi_{1}} \Gamma_{1} \chi_{1}\right)\left(\bar{f} \Gamma_{2} f\right), \\
& \frac{1}{\Lambda_{12}^{2}}\left(\overline{\chi_{1}} \Gamma_{1} \chi_{2}\right)\left(\bar{f} \Gamma_{2} f\right)+\text { h.c. } \\
& \frac{1}{\Lambda_{22}^{2}}\left(\overline{\chi_{2}} \Gamma_{1} \chi_{2}\right)\left(\bar{f} \Gamma_{2} f\right),
\end{aligned}
$$

where $\chi_{1}$ and $\chi_{2}$ are Dirac fermions. In the examples we present below we adopt vector operators with $\Gamma_{1}=$ $\Gamma_{2}=\gamma_{\mu}$. We assume throughout this work that $\chi_{1}$ and $\chi_{2}$ have similar masses, and take $\Lambda_{11} \gg \Lambda_{12}, \Lambda_{22}$. This ensures the self-annihilation rate of $\chi_{1}$ is subdominant, and hence the co-annihilation processes truly control the relic density determination. Collider production of dark sector particles will thus be dominated by $\chi_{1} \overline{\chi_{2}}$ and $\chi_{2} \overline{\chi_{2}}$

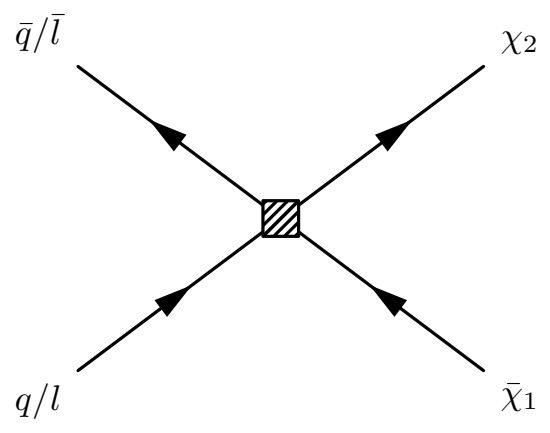

FIG. 1. Effective operators

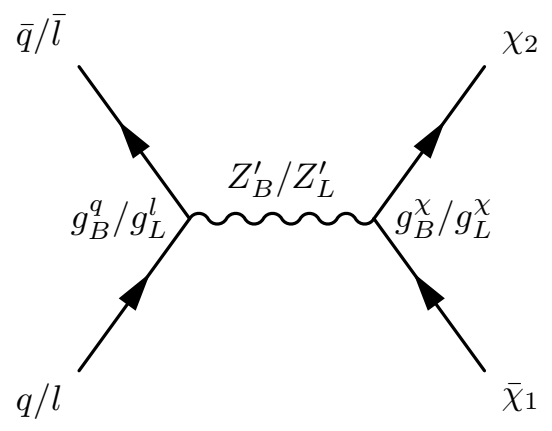

FIG. 2. UV Completion

states, with a negligible rate for pair production of the $\mathrm{DM}, \chi_{1} \overline{\chi_{1}}$.

In the limit that $\Lambda_{11} \gg \Lambda_{12}, \Lambda_{22}$, direct detection signals will be highly suppressed. Direct detection signals of the inelastic type [41, 42], $\chi_{1}+N \rightarrow \chi_{2}+N$, will not occur unless the mass splitting between $\chi_{1}$ and $\chi_{2}$ is smaller than the energy transfer in a DM-nucleus scattering interaction, $\Delta m_{\chi}=m_{\chi_{2}}-m_{\chi_{1}} \lesssim 100 \mathrm{KeV}$. This is much smaller than the mass splittings we consider. (See Ref. [43] for collider signatures of inelastic dark matter.) Hence, although direct detection provides very strong constraints in the usual self-annihilation scenario (at least for effective operators that lead to spin independent DM-nucleon scattering) this link is broken for co-annihilation. ${ }^{1}$ It is thus relic density and collider searches that provide the most relevant constraints on the co-annihilation scenario.

However, as mentioned above, an EFT description may breakdown for high energy collider experiments. Therefore, we consider a simple UV completion by introducing two neutral massive gauge bosons, $Z_{B}^{\prime}$ and $Z_{L}^{\prime}$, associated with the spontaneous breaking of the gauged symmetries $U(1)_{B}$ and $U(1)_{L}$ respectively. We assume that spectator fermions necessary to cancel the corresponding

\footnotetext{
${ }^{1}$ Importantly, because co-annihilation eliminates direct detection constraints, we encounter the interesting scenario whereby a mono-jet signal may be seen in a region of parameter space for which self-annihilating dark matter has already been ruled out.
} 
anomalies are massive enough to evade current collider constraints. The relevant interaction terms in the UVtheory are given by,

$$
\begin{aligned}
\Delta \mathcal{L}_{U V} & =g_{B}^{q} \bar{q} \gamma^{\mu} Z_{B, \mu}^{\prime} q+g_{L}^{l} \bar{l} \gamma^{\mu} Z_{L, \mu}^{\prime} l \\
& +g_{B}^{\chi}\left[\overline{\chi_{2}} \gamma^{\mu} Z_{B, \mu}^{\prime} \chi_{1}+h . c+\overline{\chi_{2}} \gamma^{\mu} Z_{B, \mu}^{\prime} \chi_{2}\right] \\
& +g_{L}^{\chi}\left[\overline{\chi_{2}} \gamma^{\mu} Z_{L, \mu}^{\prime} \chi_{1}+h . c+\overline{\chi_{2}} \gamma^{\mu} Z_{L, \mu}^{\prime} \chi_{2}\right]
\end{aligned}
$$

where $q$ and $l$ represent the SM quarks and leptons. The $Z_{B}^{\prime}\left(Z_{L}^{\prime}\right)$ gauge boson couples to quarks (leptons) with strength $g_{B}^{q}\left(g_{L}^{l}\right)$ and to dark sector particles with strength $g_{B}^{\chi}\left(g_{L}^{\chi}\right)$. Notice that in this case there are two effective scales,

$$
\Lambda_{12, B}=\Lambda_{22, B}=\frac{M_{Z_{B}^{\prime}}}{\sqrt{g_{B}^{q} g_{B}^{\chi}}}, \quad \Lambda_{12, L}=\Lambda_{22, L}=\frac{M_{Z_{L}^{\prime}}}{\sqrt{g_{L}^{l} g_{L}^{\chi}}} .
$$

The $\bar{f} f \leftrightarrow \chi_{i} \overline{\chi_{j}}$ processes in the EFT and UV complete descriptions are shown in Fig. 1 and Fig. 2, respectively. For the parameters we choose, the EFT description will be approximately valid for the relic density calculation as we describe in the next section. However, for the LHC collider phenomenology, where the momentum transfer can be large and the EFT breaks down, we use the UVcomplete theory.

\section{DARK MATTER RELIC DENSITY}

In the co-annihilation scenario we calculate the relic number density at freezeout of $n=\sum_{i} n_{\chi_{i}}$. The $\chi_{2}$ component will then decay to the stable DM particle $\chi_{1}$, to form the relic DM in the universe today. The effective annihilation cross section of the $\chi_{i}$ to SM fermions, $f$, is $[32,33]$

$$
\begin{aligned}
\sigma_{\text {eff }}=\frac{1}{\left(g_{\text {eff }} / 4\right)^{2}}[ & \sigma_{\chi_{1} \bar{\chi}_{1} \rightarrow f \bar{f}}+e^{-\Delta x}(1+\Delta)^{3 / 2} \sigma_{\chi_{1} \bar{\chi}_{2} \rightarrow f \bar{f}} \\
& \left.+e^{-2 \Delta x}(1+\Delta)^{3} \sigma_{\chi_{2} \bar{\chi}_{2} \rightarrow f \bar{f}}\right]
\end{aligned}
$$

where $x \equiv m_{\chi_{1}} / T$,

$$
\Delta \equiv \frac{m_{\chi_{2}}-m_{\chi_{1}}}{m_{\chi_{1}}}
$$

and

$$
g_{\mathrm{eff}}=4+4 e^{-\Delta x}(1+\Delta)^{3 / 2} .
$$

We are interested in parameters for which the selfannihilation cross section of $\chi_{1}, \sigma_{\chi_{1} \bar{\chi}_{1}}$ is suppressed due to the large UV cut-off $\Lambda_{11}$. Unless the mass splitting of $\chi_{1}$ and $\chi_{2}$ is extremely small, we expect the last term in Eq.(3.1) to provide a negligible contribution to $\sigma_{\text {eff }}$ due to the double exponential suppression. Working under these assumptions the co-annihilation of $\chi_{1}$ and $\chi_{2}$ dominates the dark matter depletion in the early universe. We now determine the parameter values necessary to ob- tain the observed DM relic density.

In the usual thermal freeze-out scenario we can apply a non-relativistic expansion of the annihilation cross section such that the thermal average is given by $\left\langle\sigma_{\text {eff }} v\right\rangle \approx$ $a_{\text {eff }}+6 b_{\text {eff }} / x$. The relic density is then approximated to good accuracy by [44]

$$
\Omega_{\chi_{1}} h^{2}=\frac{1.07 \times 10^{9} \mathrm{GeV}^{-1} x_{F}}{g_{*}^{1 / 2} M_{P l}\left(I_{a}+3 \frac{I_{b}}{x_{F}}\right)},
$$

where $M_{P l}=1.22 \times 10^{19} \mathrm{GeV}$ is the Planck mass, $g_{*}$ is the total number of relativistic degrees of freedom at the freeze-out temperature,

$$
I_{a}=x_{F} \int_{x_{F}}^{\infty} d x \frac{a_{e f f}}{x^{2}}, \quad I_{b}=2 x_{F}^{2} \int_{x_{F}}^{\infty} d x \frac{b_{e f f}}{x^{3}}
$$

and the freeze-out temperature is determined by,

$x_{F}=\log \left(c(c+2) \sqrt{\frac{45}{8}} \frac{4}{2 \pi^{3}} \frac{m_{\chi_{1}} M_{P l}\left(a_{e f f}+b_{e f f} / x_{F}\right)}{\sqrt{g_{e f f} x_{F}}}\right)$,

with $c \approx 1 / 2$. Thus it is clear that, in the EFT limit, the relic density $\Omega_{\chi_{1}}$ is completely determined by the parameters $m_{\chi_{1}}, \Delta$ and $\Lambda_{12}$. We implemented the model with FeynRules [45] and generated model files for MicroMEGAs [46] for the relic abundance calculation. We then performed a scan over these parameters such that a relic density in approximate accordance with the Planck results was obtained, $\Omega_{D M}=0.1187 \pm 0.0017$ [47].

In Fig. 3 we show the results of the scan for dark matter mass between $50 \mathrm{GeV}$ and $1 \mathrm{TeV}$ and $\Lambda_{12, B} / m_{\chi_{1}}$ between 2 and 6 , assuming that dark matter only couples to quarks, i.e. $\Lambda_{L} \gg \Lambda_{B} \cdot{ }^{2}$ The necessary mass splitting for successful co-annihilation is typically $\Delta \lesssim 0.3$, and in Fig. 3 we show contours corresponding to $\Delta=0.05-0.25$. For fixed $\Lambda_{12, B} / m_{\chi_{1}}$, the co-annihilation cross section decreases with increasing $m_{\chi_{1}}$, which in turn requires more efficient co-annihilation, i.e. a smaller $\Delta$. On the other hand, for fixed $m_{\chi_{1}}$, the annihilation cross section also decreases with increasing $\Lambda_{12}$, which again can be compensated with a more efficient co-annihilation.

In Fig. 3 we also indicate the parameters for which the EFT description is valid. The parameters above the solid line satisfy $\Omega_{\chi_{1}, \mathrm{UV}} / \Omega_{\chi_{1} \text {,eff }} \geq 0.8$, where $\Omega_{\chi_{1}, \mathrm{UV}}$ and $\Omega_{\chi_{1} \text {,eff }}$ are the relic densities calculated using the UV complete and EFT descriptions, respectively. We see that the EFT underestimates the relic density unless $\Lambda_{12}$ is sufficiently large, $\Lambda_{12} / m_{\chi_{1}} \gtrsim 5 .^{3}$

\footnotetext{
2 When we turn to the collider phenomenology, we shall be interested in dark matter which couples to both quarks and leptons. Thus the relic density calculations will be appropriately modified to take the additional annihilation channels into account.

${ }^{3}$ If $M_{Z_{B, L}^{\prime}} \approx \sqrt{s} \approx 2 m_{\chi_{1}}$ resonance effects become important which are not taken into account in the effective theory.
} 


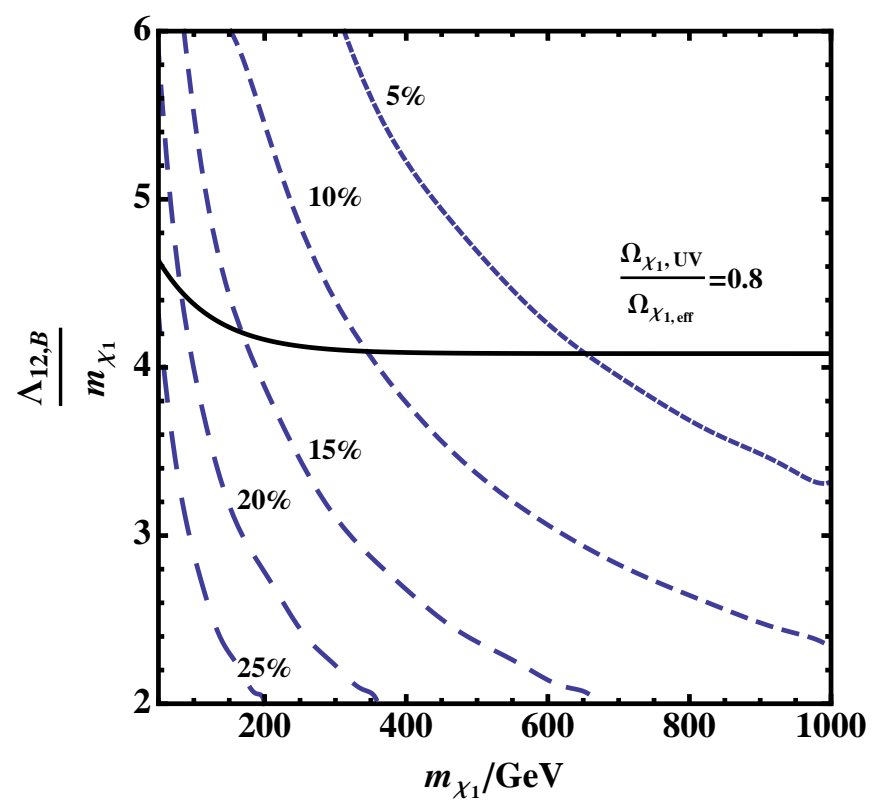

FIG. 3. Contours of the mass splitting $\Delta$ which satisfy the correct DM abundance in the EFT approximation. Contours are shown with dashed lines for $\Delta=0.05,0.1,0.15,0.2$ and 0.25 , as indicated. The solid line represents $\Omega_{\chi_{1}, \mathrm{UV}} / \Omega_{\chi_{1} \text {,eff }}=$ 0.8 , where $\Omega_{\chi_{1}, \mathrm{UV}}$ is calculated taking $g_{B}^{q}=g_{B}^{\chi}=1$. The EFT provides an adequate description for parameters above this line.

In section $\mathrm{V}$ we shall consider collider signatures for parameters consistent with the the DM relic density. Note that because $\chi_{1} \bar{\chi}_{2}$ co-annihilation makes an exponentially suppressed contribution to $\sigma_{\text {eff }}$ (see Eq. (3.1)), we require larger coupling constants than for the standard case of a single dark sector species. Correspondingly, the collider production rates are larger.

\section{COUPLINGS CONSTRAINTS FROM DI-JET AND DI-LEPTON SEARCHES}

As with many dark matter models, in addition to searches for dark matter production, important constraints arise from direct production of the particles which mediate the DM-SM interaction. In our model these mediators are the $Z_{L}^{\prime}$ and $Z_{B}^{\prime}$ particles. We are thus led to consider di-jet and di-lepton signals with no $E_{T}$, mediated by $Z^{\prime}$ exchange.

In an EFT description the relevant four-fermion effective operators are

$$
\frac{1}{\Lambda_{l}^{2}} \bar{l} \gamma^{\mu} l \bar{l} \gamma_{\mu} l, \quad \frac{1}{\Lambda_{q}^{2}} \bar{q} \gamma^{\mu} q \bar{q} \gamma_{\mu} q
$$

where $\Lambda_{l}=M_{Z_{L}^{\prime}} /\left|g_{Z}^{l}\right|$ and $\Lambda_{q}=M_{Z_{B}^{\prime}} /\left|g_{B}^{q}\right|$. There have been several experiments that constrain the allowed parameter space of operators of the form of Eq. (4.1). For example, $\Lambda_{l}$ is constrained by dilepton searches at LEP
II. For $m_{Z_{L}^{\prime}}>200 \mathrm{GeV}$, dilepton production through an off-shell mediator results in the bound $g_{L}^{l} \lesssim g_{L, \max }^{l} \equiv$ $0.044 \times\left(m_{Z_{L}^{\prime}} / 200 \mathrm{GeV}\right)[48,49]$.

On the other hand, the $Z_{B}^{\prime}$ parameters are constrained by di-jet searches at hadron colliders such as UA2 at the CERN SPS collider, CDF at the Tevatron, and ATLAS and CMS at the LHC. For example, Ref. [50] reports limits on the $g_{Z_{B}^{\prime}}-M_{Z_{B}^{\prime}}$ coupling-mass plane, arising from di-jet resonance searches. (Note that di-jet resonances, corresponding to on-shell $Z_{B}^{\prime}$ production, cannot be described in terms of an EFT.) However, these limits assume the new $Z_{B}^{\prime}$ boson decays only to SM particles. If the $Z_{B}^{\prime}$ boson can also decay to dark sector particles, the limits from the di-jet resonances searches are weakened because the on-shell $Z^{\prime}$ can now decay to invisible final states. If the $Z_{B}^{\prime}$-width is narrow, the s-channel production factorizes from the decay, $\sigma(j j)=\sigma_{Z^{\prime}} \times \operatorname{Br}\left(Z^{\prime} \rightarrow\right.$ $j j)$. Although the di-jet branching ratio will be reduced by the presence of other decay channels, we can safely assume that the di-jet acceptance is not altered. Therefore, we see that the effect of the additional $Z^{\prime}$ decay modes is to increase the maximum allowed coupling to quarks by a factor of $1 / \sqrt{\mathrm{Br}}$, namely

$$
\begin{aligned}
g_{B, \text { max }}^{q} & =g_{B}^{q} \frac{1}{\sqrt{B r\left(Z_{B}^{\prime} \rightarrow j j\right)}}, \\
& =g_{B}^{q}\left[1+\frac{\Gamma\left(Z_{B}^{\prime} \rightarrow \chi \bar{\chi}\right)}{\Gamma\left(Z_{B}^{\prime} \rightarrow j j\right)}\right]^{1 / 2}, \\
& \approx g_{B}^{q}\left[1+\frac{r_{B}^{2}}{N_{c} N_{f}}\left(1+2 \frac{m_{\chi_{1}}^{2}}{M_{Z_{B}^{\prime}}^{2}}\right) \sqrt{\left.1-\frac{4 m_{\chi_{1}}^{2}}{M_{Z_{B}^{\prime}}^{2}}\right]^{1 / 2}}\right.
\end{aligned}
$$

where $r_{B}=g_{B}^{\chi} / g_{B}^{q}$ is the coupling ratio, $N_{c}$ and $N_{f}$ are the number of color and flavour states the $Z_{B}^{\prime}$ can decay into, and we have neglected SM-fermion masses since $m_{f, S M} \ll m_{\chi_{1}}, M_{Z_{B}^{\prime}}$.

We shall choose example parameters that are consistent with the pure di-jet and di-lepton constraints, and which also produce the correct DM relic density. For this purpose, it is useful to write the couplings $g_{B}^{q}$ and $g_{L}^{l}$ as functions of the coupling ratios $r_{B}$ and $r_{L}$ respectively,

$$
g_{(B, L)}^{(q, l)}=\frac{M_{Z_{B, L}^{\prime}}}{\Lambda_{12,(B, L)} r_{B, L}^{1 / 2}} .
$$

In order to maximise the possible signals, we choose couplings that are close to, but do not saturate, the current bounds. Specifically, we take $g_{L, \max }^{l}-g_{L}^{l}=0.01$ and $g_{B, \text { max }}^{q}-g_{B}^{q}=0.1$. We will divide our analyses in two groups. In the first group, we only allow $U(1)_{L}$ and $U(1)_{B}$ couplings of the $Z^{\prime}$ s to SM particles and hidden sector particles that are below 1 . We refer to this group as the weak coupling group. The second group consists of points in parameter space where the $U(1)_{L}$ and $U(1)_{B}$ $Z^{\prime}$ couplings to hidden sector particles, $g_{B}^{\chi}$ and $g_{L}^{\chi}$, are allowed to be larger than 1 . We refer to this group as the strong coupling group. For the weak coupling group we 
fixed the $Z_{L}^{\prime}$ mass to be $M_{Z_{L}^{\prime}}=250 \mathrm{GeV}$, which allows for smaller $g_{L}^{\chi}$-couplings, while for the strong coupling group we choose $M_{Z_{L}^{\prime}}=550 \mathrm{GeV}$. Using Eq. (4.3), we can then obtain masses and couplings that satisfy both the relic density and collider constraints. Some representative parameters are shown in Table I.

\section{COLLIDER PHENOMENOLOGY}

\section{A. Signal processes}

We now examine the new LHC signals that arise from $\bar{\chi}_{1} \chi_{2}$ production followed by the decay $\chi_{2} \rightarrow \chi_{1}+S M$. The lowest order $\bar{\chi}_{1} \chi_{2}$ production process at the LHC is

$$
p p \rightarrow \bar{\chi}_{1} \chi_{2} \rightarrow \bar{\chi}_{1} \chi_{1}+S M .
$$

In this case the only visible particles in the final state are the SM states $(\bar{q} q$ or $\bar{l} l)$ produced through the decay of the $\chi_{2}$. The signal for this process is thus di-leptons or di-jets plus $\mathscr{E}_{T}$. However, given that successful co-annihilation requires a relatively small mass difference between $\chi_{2}$ and $\chi_{1}, \Delta m \lesssim 0.3 m_{\chi_{1}}$, the SM particles have relatively soft energies and the remnant hidden particles $\chi_{1}$ and $\bar{\chi}_{1}$ are approximately back-to-back, leading to a small net $E_{T}$. This is crucial for the possible detectability of this signal. At a hadron collider the irreducible $Z+$ jets background, with soft jets from the underlying QCD processes, provides an enormous number of events with dileptons or dijets and small $E_{T}$, even when demanding a $Z$-veto by rejecting events whose dilepton or dijet invariant mass is close to the $Z$ boson pole mass. The process in Eq.(5.1) would be hidden by this large background at the LHC. ${ }^{4}$.

In order to have any chance of observing $\bar{\chi}_{1} \chi_{2}$ production in a hadron collider, it becomes imperative to look for processes that breaks the back-to-back alignment between the hidden particles and therefore lead to a substantial $E_{T}$. This alignment rupture is in fact produced by a hard jet in the form of initial state radiation (ISR), as is the case for monojet searches. In this case the hidden particles recoil against the hard jet and a large amount of $E_{T}$ can be produced. Therefore, the process of interest at the LHC is

$$
p p \rightarrow \overline{\chi_{1}} \chi_{2}+j \rightarrow \bar{\chi}_{1} \chi_{1}+S M+j,
$$

where the ISR jet is hard. Given that we have two mediators, the possible $\chi_{2}$ decay chains are

$$
\begin{aligned}
& \chi_{2} \rightarrow \chi_{1}+Z_{B}^{\prime *} \rightarrow \chi_{1}+q+\bar{q}, \\
& \chi_{2} \rightarrow \chi_{1}+Z_{L}^{\prime *} \rightarrow \chi_{1}+l+\bar{l},
\end{aligned}
$$

\footnotetext{
${ }^{4}$ In a lepton collider such as the ILC it is conceivable that the dijet or dilepton plus $E_{T}^{\prime}$ signal may be discoverable due to the absence of underlying QCD process, making it a cleaner environment.
}

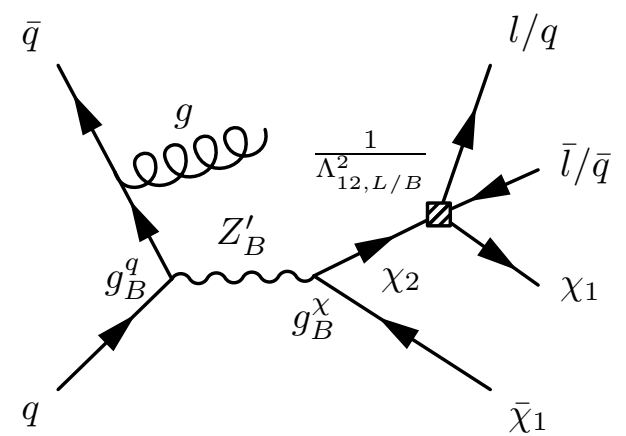

FIG. 4. Contribution to the jet plus $E_{T}$ plus dilepton (or diquark) signal at the LHC. Additional diagrams with other initial state partons are not shown.

and hence the final states will be a hard jet from ISR in addition to $E_{T}$ and either di-jets or di-leptons. A representative diagram is shown in Fig. 4. Again, due to the small mass splitting $\Delta m$, the energy of the di-jets or di-leptons will be relatively low. ${ }^{5}$. As it would be challenging to identify di-jet signals with low $p_{T}$, due to the large QCD backgrounds encountered at the LHC, we shall concentrate on the di-lepton channel.

The production cross sections of $\overline{\chi_{1}} \chi_{2}$ plus $\overline{\chi_{2}} \chi_{1}$ are shown in Table I. Also shown are the cross sections for $\overline{\chi_{1}} \chi_{1} l^{+} l^{-}$, which are related to the previous cross sections by the $\chi_{2}$ branching ratio to leptons, which is typically $O(10 \%)$ for our parameters.

\section{B. Backgrounds and event selection at the LHC}

We now perform a detailed calculation of the signal and background for the process $p p \rightarrow \bar{\chi}_{1} \chi_{2} j \rightarrow \bar{\chi}_{1} \chi_{1} j l^{+} l^{-}$, which is observed as a hard jet, large $E_{T}$, and two same flavour opposite-sign leptons $\left(l^{+} l^{-}\right)$, where by leptons we mean light leptons $l=\{e, \mu\}$.

We use MadGraph 5 [51] to simulate signal and all background events. The built-in Pythia [52] and Delphes [53] in MadGraph 5 are used to simulate the hadronization, showering and detector effects. We adopt the MLM jet matching algorithm and set xqcut $=15 \mathrm{GeV}$. We perform all analysis with MadAnalysis 5 [54]. Our intention is not to optimize the analysis to set precise constraints, but to instead illustrate the potential for these dark matter models to be constrained by the LHC experiments, and indicate which regions of parameter space can be probed with forthcoming LHC data.

As mentioned earlier, to overcome the large background from $Z\left(\rightarrow l^{+} l^{-}\right)+$jets we demand a hard jet and

\footnotetext{
${ }^{5}$ Note that for the decay $\chi_{2} \rightarrow \chi_{1}+S M$, the small $\chi_{2}-\chi_{1}$ mass difference implies that the $Z^{\prime}$ propagator has a low momentum transfer and thus the EFT description will be valid.
} 


\begin{tabular}{|c||c|c|c|c|c|c|c|c|c|c|c|c|c|}
\hline example & $\begin{array}{c}m_{\chi_{1}} \\
(\mathrm{GeV})\end{array}$ & $\begin{array}{c}m_{\chi_{2}} \\
(\mathrm{GeV})\end{array}$ & $\begin{array}{c}m_{Z_{B}^{\prime}} \\
(\mathrm{GeV})\end{array}$ & $g_{B}^{q}$ & $g_{B}^{\chi}$ & $\begin{array}{c}m_{Z_{L}^{\prime}} \\
(\mathrm{GeV})\end{array}$ & $g_{L}^{l}$ & $g_{L}^{\chi}$ & $\begin{array}{c}\Lambda_{l} \\
(\mathrm{GeV})\end{array}$ & $\begin{array}{c}p_{T}\left(l_{1}\right) \\
(\mathrm{GeV})\end{array}$ & $\begin{array}{c}M\left(l^{+} l^{-}\right) \\
(\mathrm{GeV})\end{array}$ & $\begin{array}{c}\sigma_{p p \rightarrow \bar{\chi}_{i} \chi_{j}} \\
14 \mathrm{TeV}(\mathrm{fb})\end{array}$ & $\begin{array}{c}\sigma_{p p \rightarrow \bar{\chi}_{1} \chi_{1} l^{+} l^{-}} \\
14 \mathrm{TeV}(\mathrm{fb})\end{array}$ \\
\hline \hline 1. & 250 & 270 & 525 & 0.15 & 0.80 & 250 & 0.045 & 0.66 & 1450 & $<30$ & $<20$ & 6597 & 552 \\
\hline 2. & 300 & 321 & 625 & 0.14 & 0.89 & 250 & 0.045 & 0.53 & 1620 & $<30$ & $<20$ & 3694 & 376 \\
\hline 3. & 400 & 420 & 825 & 0.18 & 0.68 & 250 & 0.045 & 0.32 & 2080 & $<30$ & $<20$ & 905 & 102 \\
\hline 4. & 600 & 612 & 1700 & 0.23 & 0.98 & 250 & 0.045 & 0.15 & 3000 & $<20$ & $<15$ & 442 & 52 \\
\hline \hline 5. & 400 & 432 & 1375 & 0.21 & 2.2 & 550 & 0.11 & 0.8 & 1840 & $<60$ & $<30$ & 2285 & 186 \\
\hline 6. & 500 & 530 & 1500 & 0.18 & 1.83 & 550 & 0.11 & 0.52 & 2300 & $<60$ & $<30$ & 1103 & 104 \\
\hline 7. & 600 & 630 & 1475 & 0.16 & 1.61 & 550 & 0.11 & 0.36 & 2760 & $<40$ & $<30$ & 852 & 70 \\
\hline 8. & 700 & 728 & 1425 & 0.12 & 1.51 & 550 & 0.11 & 0.26 & 3220 & $<30$ & $<30$ & 193 & 16 \\
\hline
\end{tabular}

TABLE I. Coupling parameters, cross sections, and kinematic cuts for eight sets of example parameters. The second and the third column are the masses of the two co-annihilating particles, while the fourth to the ninth columns show the $Z_{B}^{\prime}$ and $Z_{L}^{\prime}$ mass and coupling parameters. We also show the cuts on the leading lepton- $p_{T}$, the invariant mass of the dileptons and the $\Delta R$ separation between the leading lepton and the leading jet. The last column is the cross section for $p p \rightarrow \chi_{i} \bar{\chi}_{j}+j \rightarrow \chi_{1} \bar{\chi}_{1} l^{+} l^{-}+j$ at the $14 \mathrm{TeV}$ LHC.

\begin{tabular}{|c|c|c|c|}
\hline Cuts & Signal (S) & Background (B) & Significance $(S / \sqrt{S+\Delta B})$ \\
\hline $\begin{array}{c}p_{T}(l)>10 \mathrm{GeV},\left|\eta_{l e p}\right|<2.5, \\
\Delta R_{l^{+} l^{-}}>0.4, \Delta R_{l j}>0.4 \\
M\left(l^{+} l^{-}\right)>5 \mathrm{GeV}\end{array}$ & 7520 & 1062935 & 0.10 \\
\hline$p_{T}\left(j_{1}\right)>150 \mathrm{GeV}$ & 1650 & 428354 & 0.04 \\
\hline$E_{T}>120 \mathrm{GeV}$ & 1079 & 22090 & 0.61 \\
\hline$M\left(l^{+} l^{-}\right)<20 \mathrm{GeV}$ & 55 & 85 & 3.8 \\
\hline$N(b)=0$ & 53 & 38 & 5.2 \\
\hline$p_{T}\left(l_{1}\right)<30$ & 52 & 14 & 6.3 \\
\hline
\end{tabular}

TABLE II. The cut-flow chart for example \#2 with $\mathcal{L}=20 \mathrm{fb}^{-1}$. The cuts are sequential.

large $E_{T}$, which makes this background negligible [55]. Specifically, we choose $p_{T}(j)>150 \mathrm{GeV}$, and $E_{T}>120$ $\mathrm{GeV}$. We verified this in our analysis as can be seen in Figs. 5-8 where one can see that the combination of the requirements of a hard jet and large $E_{T}$ make this background negligible. Furthermore, we impose a minimum cut on the invariant mass of the dileptons $M\left(l^{+} l^{-}\right)>5$ $\mathrm{GeV}$ in order to veto background events from $J / \psi$ decays.

Another important background is the top pair production which subsequently decays via $t \bar{t} \rightarrow b \bar{b} l^{+} l^{-} \nu_{l} \bar{\nu}_{l}$. We can eliminate most of the $t \bar{t}$ background by demanding the leading lepton have $p_{T}<30-60 \mathrm{GeV}$ and that the $b$-jet multiplicity be zero.

The main SM backgrounds remaining are diboson pair production, $Z Z \rightarrow l^{+} l^{-} \bar{\nu}_{l} \nu_{l} / l^{+} l^{-} j j, W W \rightarrow l^{+} l^{-} \bar{\nu}_{l} \nu_{l}$ and $W^{ \pm} Z \rightarrow l^{+} l^{-} l^{ \pm} \nu$, where the leptons are of the same flavour and in the last process one of the leptons is missed. The hard jet can either come from the process itself or the underlying events. Since the diboson backgrounds are all electroweak processes, they have much smaller cross section compared with the $t \bar{t}$ and $Z+j e t s$ background and are thus subdominant.
We trigger our events by demanding $E_{T}>120 \mathrm{GeV}$ and $p_{T}(j)>150 \mathrm{GeV}$. We adopt conservative requirements for the charged leptons, $p_{T}(l)>10 \mathrm{GeV},|\eta|<2.5$, $\Delta R\left(l^{+}, l^{-}\right)>0.4$ and $\Delta R(l, j)>0.4$, where $\Delta R$ is the separation in the $\eta-\phi$ plane such that the leptons are isolated ${ }^{6}$. We also demand the jet to have $|\eta|<5$. The remaining cuts were chosen to suppress as much background as possible without diminishing the signal. The kinematic observables upon which we impose cuts are: the invariant mass of the leptons $M\left(l^{+} l^{-}\right)$and $p_{T}$ of the leading lepton. We also veto the events with nonzero $b$ jet multiplicity. When considering high luminosities, in order to further reduce the background, we also imposed an upper bound on the leading lepton, $p_{T}\left(l_{1}\right)$. We list in Table I the corresponding cuts for the LHC at $14 \mathrm{TeV}$, for the sample DM parameters considered. In Table II we show, for the \#2 example parameters, how each cut

\footnotetext{
${ }^{6}$ In principle, because we trigger on the $E_{T}>$ and the jet $p_{T}$, we could include lower momentum leptons, $p_{T}(l) \gtrsim 6 \mathrm{GeV}$, which would further boost our signal.
} 
TABLE III. Signal and Background after cuts for the example parameters.

\begin{tabular}{|c|c|c|c|c|c|}
\hline Example \# & $\mathcal{L}$ at $s=14 \mathrm{TeV}$ & Signal $(\mathrm{S})$ & Background $(\mathrm{B})$ & $S / B$ & $S / \sqrt{S+\Delta B}$ \\
\hline \hline 1. & $\mathcal{L}=20 \mathrm{fb}^{-1}$ & 67 & 14 & 4.8 & 7.4 \\
\hline 2. & $\mathcal{L}=20 \mathrm{fb}^{-1}$ & 52 & 14 & 3.7 & 6.3 \\
\hline 3. & $\mathcal{L}=200 \mathrm{fb}^{-1}$ & 106 & 137 & 0.77 & 5.1 \\
\hline 4. & $\mathcal{L}=300 \mathrm{fb}^{-1}$ & 23 & 41 & 0.56 & 2.6 \\
\hline 5. & $\mathcal{L}=20 \mathrm{fb}^{-1}$ & 159 & 93 & 1.7 & 8.6 \\
\hline 6. & $\mathcal{L}=20 \mathrm{fb}^{-1}$ & 81 & 93 & 0.87 & 5.0 \\
\hline 7. & $\mathcal{L}=20 \mathrm{fb}^{-1}$ & 51 & 63 & 0.80 & 4.1 \\
\hline 8. & $\mathcal{L}=300 \mathrm{fb}^{-1}$ & 114 & 538 & 0.21 & 1.9 \\
\hline
\end{tabular}

affects signal and background at $14 \mathrm{TeV}$ and a luminosity of $\mathcal{L}=20 \mathrm{fb}^{-1}$.

In Figs. 5, 6, 7 and 8 we show histograms of the kinematic variables after all cuts except the one on the kinematic variable itself, for the example \#2 at $\mathcal{L}=20 \mathrm{fb}^{-1}$ and example $\# 8$ at $\mathcal{L}=300 \mathrm{fb}^{-} 1$. Note from Fig. 5 that despite demanding a large $E_{T}$, there are still a sizable number of events due to the balance between the ISR jet $p_{T}$ (which tends to be hard) and the $p_{T}$ of the $\chi$. Due to the small mass difference $\Delta m \ll m_{\chi_{1}} \approx m_{\chi_{2}}$, the lepton momentum is typically soft (see Fig. 7). For the same reason the invariant mass of the dileptons tends to be comparable to $\Delta m$, as seen in Fig. 8 .

We define our signal significance via the ratio $S / \sqrt{S+\Delta B}$ with

$$
\Delta B=\sqrt{\sum_{i}\left[B_{i}+\left(\beta_{i} B_{i}\right)^{2}\right]}
$$

where $\beta_{i}$ is set to be $10 \%$ to account for the systematic error. In Table III we list the signal (S), background (B), the ratio of signal over background and the significances for different example parameters, considering the minimal luminosity $\left(\mathcal{L} \lesssim 300 \mathrm{fb}^{-1}\right)$ needed to obtain a significance of order 5 (or the largest possible for $\mathcal{L}=300$ $\left.\mathrm{fb}^{-1}\right)$.

Based on the sensitivities shown in Table III, we conclude that the $14 \mathrm{TeV}$ LHC does have the capability to identify a significant signal. For the weak coupling group (examples \#1-4), dark matter masses up to $\sim 400 \mathrm{GeV}$ would be probed in the first $200 \mathrm{fb}^{-1}$ of luminosity at the $14 \mathrm{TeV}$ LHC, while it may be possible to get a strong hint for a dark matter mass of $600 \mathrm{GeV}$ with $\mathcal{L}=300$ $\mathrm{fb}^{-1}$. For the strong coupling group (examples \#5-8), dark matter masses $m_{\chi} \lesssim 700 \mathrm{GeV}$ would be probed in the first $300 \mathrm{fb}^{-1}$ of luminosity at the $14 \mathrm{TeV}$ LHC. For masses $m_{\chi_{1}} \gtrsim 750 \mathrm{GeV}$, the production cross-sections become too small and therefore larger luminosities are needed. It is, however, important to stress that scenarios with dark matter masses below $\sim 250 \mathrm{GeV}$ are already constrained by LHC measurements and even with $20 \mathrm{fb}^{-1}$ of luminosity we should be able to detect (or rule out) scenarios with dark matter masses below $300 \mathrm{GeV}$.

Note that all our example parameters satisfy the relic density constraint, in the region where the EFT is valid. For sufficiently large masses $(\gtrsim 1 \mathrm{TeV}$ ) and reasonable choices for the coupling constants, the correct relic density cannot be explained unless we work in a region of parameter space for which the EFT is not valid, for instance, close to the resonance at $2 m_{\chi}=M_{Z}^{\prime}$. Of course, if we relax the relic density requirements, a wider range of collider cross sections are possible.

\section{Other signals}

The co-annihilation model will also lead to mono-jet plus $E_{T}$ signals. These arise from the invisible decays of the $\chi_{2}$ to neutrinos, or decays to quarks or leptons which are too soft to be identified. Both search strategies (mono-jets plus $E_{T}$ and monojets plus dileptons plus $\mathscr{E}_{T}$ ) provide complementary information in the hunt for DM at the LHC. The latest CMS mono-jet analysis [56] places the constraint $\Lambda_{12, B} \gtrsim 900 \mathrm{GeV}$ for the DM masses we consider in Table I, and all our example parameters satisfy this bound. However, to properly determine the mono-jet limits on our model, the process should be simulated using the UV-complete theory. Note, however, that Ref. [57, 58] compared monojet plus $E_{T}$ constraints, with di-jet constraints of the type discussed in section IV, for models in which DM interactions are mediated by light Z' bosons. The di-jets analyses were found to usually provide the more stringent constraints.

Finally, the possibility of pair production of $\bar{\chi}_{2} \chi_{2}$ presents further interesting signals. The process $p p \rightarrow$ $\bar{\chi}_{2} \chi_{2} \rightarrow l^{+} l^{-} l^{\prime+} l^{\prime-}$ results in two pairs of opposite signleptons, plus $E_{T}$. If we assume equal $\chi_{1}-\chi_{2}$ and $\chi_{2}-\chi_{2}$ couplings, as in Eq. (2.4), the cross section for $\bar{\chi}_{2} \chi_{2}$ production will be comparable to that for $\bar{\chi}_{1} \chi_{2}$. From the relic density point of view, the $\bar{\chi}_{2} \chi_{2}$ annihilation channel is doubly exponentially suppressed and therefore we expect a marginal contribution to the effective annihilation cross-section of Eq. (3.1). In this sense, the link between the relic density constraint and the strength of 

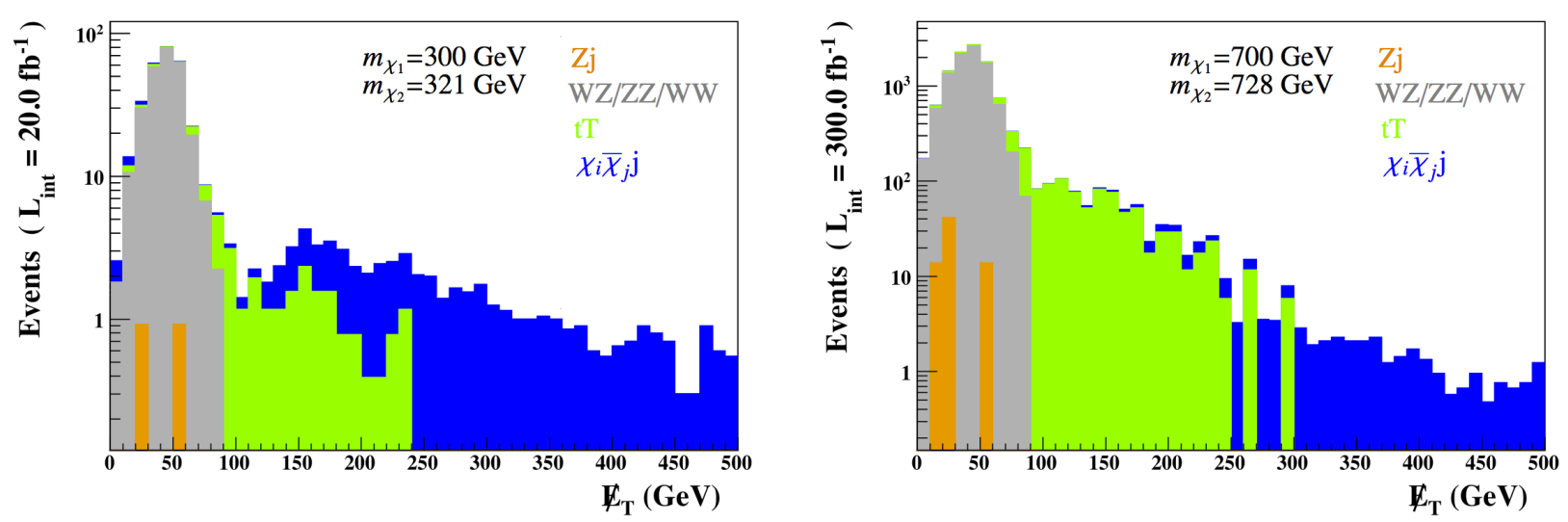

FIG. 5. Histograms of the $E_{T}$ for example \#2 (left) and \#8(right) with all the cuts applied except the one on $E_{T}$, $E_{T}>120 \mathrm{GeV}$.
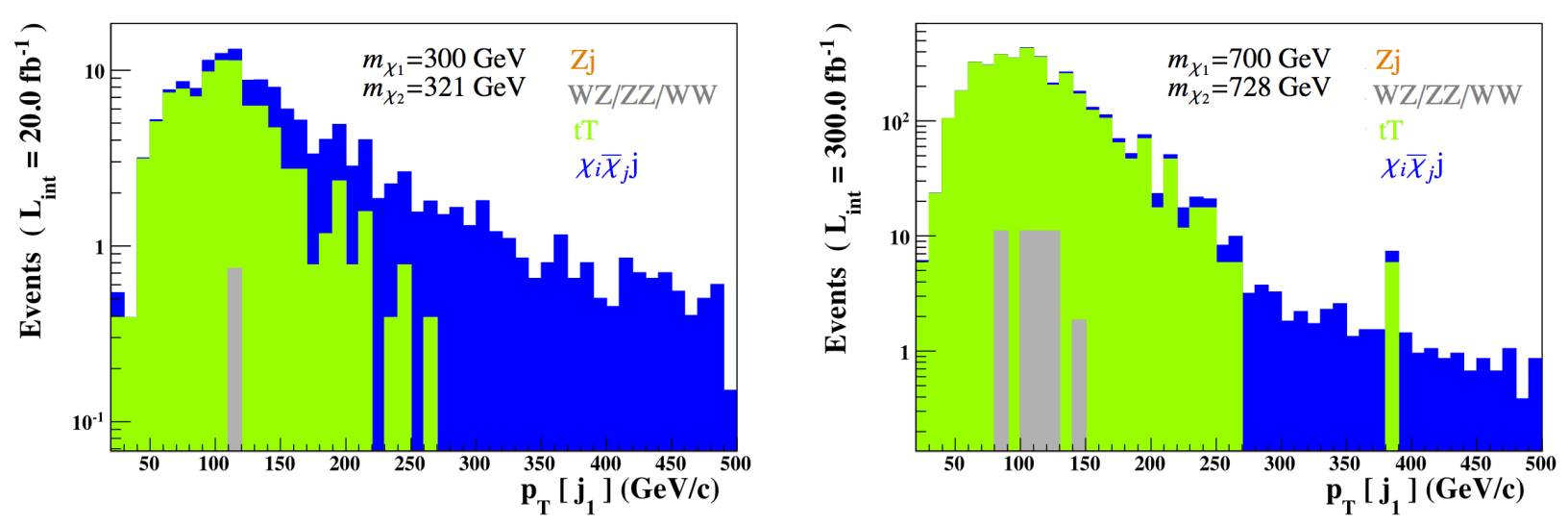

FIG. 6. Histograms of the leading jet- $p_{T}$ for example \#2 (left) and \#8(right) with all the cuts applied except the one on the leading jet- $p_{T}, p_{T}\left(j_{1}\right)>150 \mathrm{GeV}$.

a collider signal is much less direct. This type of signal is already being analysed by ATLAS [59] and, although the background is smaller than the one for a single pair of opposite-sign same-flavour leptons plus $\mathscr{E}_{T}$, it is still necessary to require a sizeable $E_{T}$ in order to suppress SM backgrounds, $E_{T} \gtrsim 50 \mathrm{GeV}$. To obtain a sufficiently large $E_{T}$, one could again require an additional ISR jet, and thus consider $l^{+} l^{-} l^{+} l^{\prime-}+$ jet $+E_{T}$. The crosssection for this type of signal can be roughly estimated as $\sigma\left(l^{+} l^{-} l^{\prime+} l^{--}+j+\chi_{1} \bar{\chi}_{1}\right) \sim(1 / 10) \times \sigma\left(l^{+} l^{-}+j+\chi_{1} \bar{\chi}_{1}\right)$. Using the parameter examples in Table I, we expect a non-negligible signal for dark matter masses up to about $600 \mathrm{GeV}$. We leave a proper analysis of this interesting signal for future work.

\section{CONCLUSION}

We have examined a scenario in which the dark sector contains two nearly degenerate particles: the DM candidate $\chi_{1}$, and a slightly heavier state $\chi_{2}$. In this scenario, the relic DM density is determined by co-annihilation processes such as $\chi_{1}+\overline{\chi_{2}} \rightarrow S M$, while direct and indi- rect detection processes are highly suppressed, because they involve $\chi_{1}$ alone. For standard self-annihilating WIMPs, there is some tension between the size of the couplings needed to obtain the correct relic abundance, and those necessary to account for the non-observation of signals in direct detection experiments. The co-annihilation scenario eliminates this incompatibility.

We described the interaction of $\chi_{1}$ and $\chi_{2}$ with SM particles, by generalising the standard EFT description. The co-annihilation model offers interesting new collider signals: In addition to the standard mono-jet + missing $E_{T}$ type of DM process, new signals arise due to $\chi_{1} \overline{\chi_{2}}$ production followed by the decay $\chi_{2} \rightarrow \chi_{1} l^{+} l^{-}$or $\chi_{2} \rightarrow \chi_{1} q \bar{q}$. We have simulated signal and background for the $l^{+} l^{-}+$jet + missing $E_{T}$ process, for parameters which correctly reproduce the DM relic density, and demonstrated that the LHC has the potential to identify these signals with forthcoming data. Of course, if dark matter is discovered at colliders, signals in multiple channels would assist in uncovering its true nature, and the new processes studied here would provide important complementary information to the standard monojet type searches. 

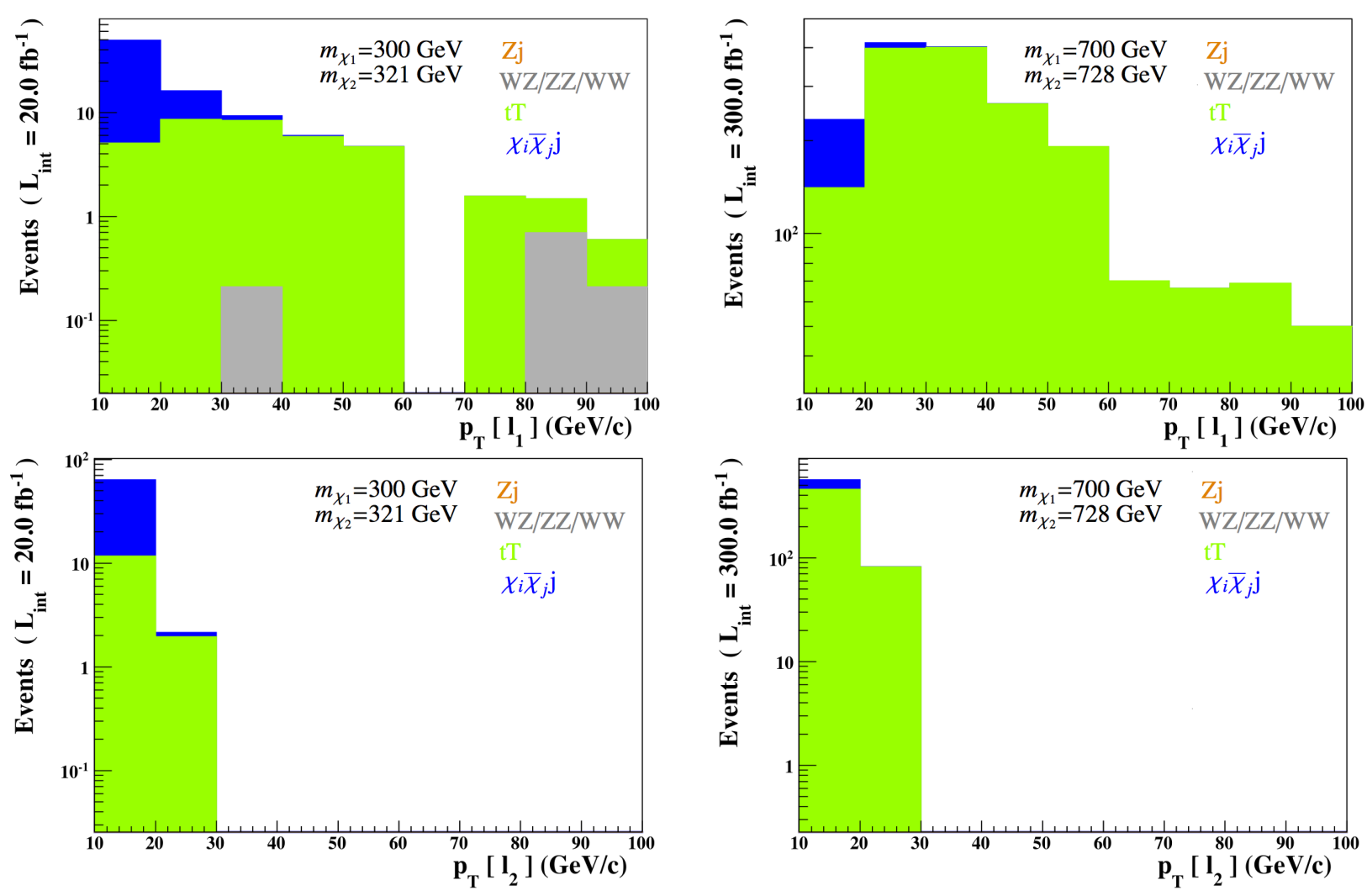

FIG. 7. Top: histograms of the leading lepton- $p_{T}$ for example \#2 (left) and \#8 (right) with all cuts applied except the ones on the leading lepton- $p_{T}, 10 \mathrm{GeV}<\mathrm{p}_{\mathrm{T}}\left(\mathrm{l}_{1}\right)<30 \mathrm{GeV}$ for both \#2 and \#8. Bottom: histograms of the second leading lepton- $p_{T}$ for example \#2 (left) and \#8 (right) with all the cuts applied.
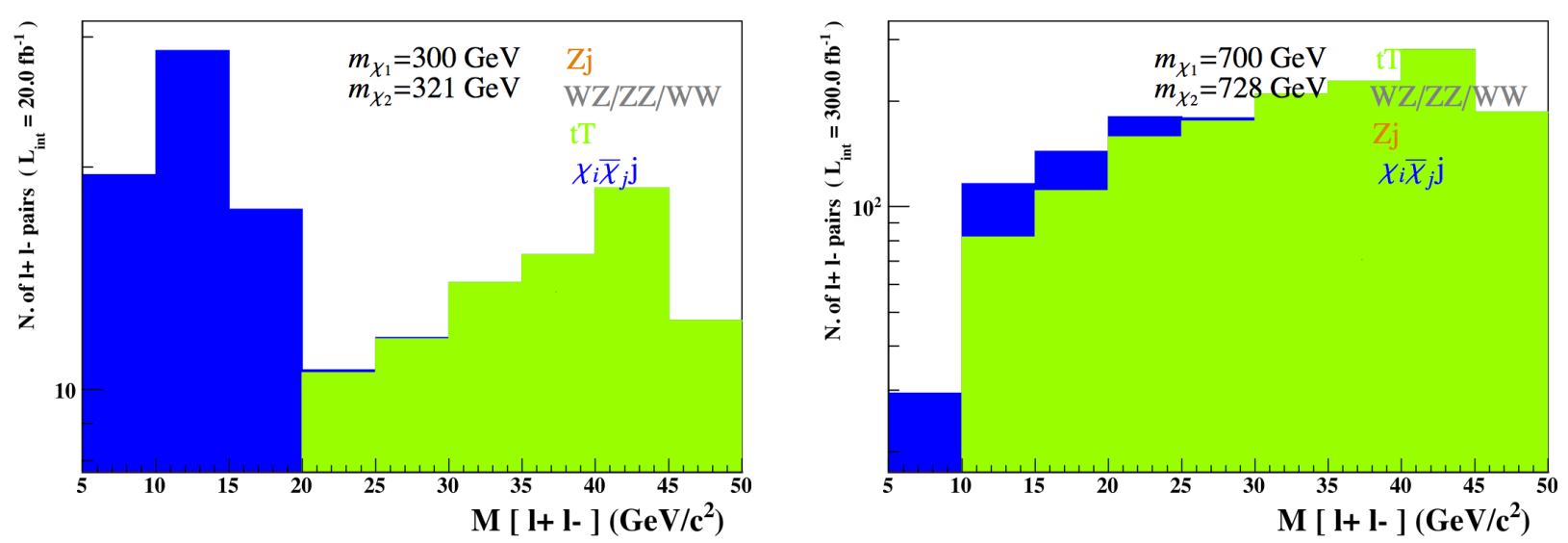

FIG. 8. Histograms of the invariant dilepton mass for example \#2 (left) and \#8 (right) with all cuts applied except the one on the invariant dilepton mass, $5 \mathrm{GeV}<\mathrm{M}(\mathrm{l}+, \mathrm{l}-)<20 \mathrm{GeV}$ for \#2 (left) and $5 \mathrm{GeV}<\mathrm{M}(\mathrm{l}+, 1-)<30 \mathrm{GeV}$ for \#8 (right).

\section{ACKNOWLEDGEMENTS}

We thank Tony Limosani, Martin White, and especially to Lei $\mathrm{Wu}$ and Brian Petersen for helpful comments and advice. We are also indebted to Heather Logan for comments and for pointing out an important error in an earlier version of this manuscript. NFB, YC and ADM were supported by the Australian Research Council.
[1] R. Agnese et al. (CDMS Collaboration), Phys.Rev.Lett. 111, 251301 (2013), 1304.4279.
[2] C. Aalseth et al. (CoGeNT Collaboration), Phys.Rev. D88, 012002 (2013), 1208.5737. 
[3] G. Angloher, M. Bauer, I. Bavykina, A. Bento, C. Bucci, et al., Eur.Phys.J. C72, 1971 (2012), 1109.0702.

[4] C. Savage, G. Gelmini, P. Gondolo, and K. Freese, JCAP 0904, 010 (2009), 0808.3607.

[5] Z. Ahmed et al. (CDMS-II Collaboration), Science 327, 1619 (2010), 0912.3592.

[6] Z. Ahmed et al. (CDMS-II Collaboration), Phys.Rev.Lett. 106, 131302 (2011), 1011.2482.

[7] R. Agnese et al. (SuperCDMSSoudan Collaboration), Phys.Rev.Lett. 112, 041302 (2014), 1309.3259.

[8] D. Akerib et al. (LUX Collaboration) (2013), 1310.8214.

[9] J. Angle et al. (XENON10 Collaboration), Phys.Rev.Lett. 107, 051301 (2011), 1104.3088.

[10] The ATLAS collaboration (ATLAS Collaboration) (2012).

[11] G. Aad et al. (ATLAS Collaboration), JHEP 1304, 075 (2013), 1210.4491.

[12] S. Chatrchyan et al. (CMS Collaboration), Phys.Rev.Lett. 108, 261803 (2012), 1204.0821.

[13] S. Chatrchyan et al. (CMS Collaboration), JHEP 1209, 094 (2012), 1206.5663.

[14] C. Collaboration (CMS Collaboration) (2013).

[15] O. Adriani et al. (PAMELA Collaboration), Nature 458, 607 (2009), 0810.4995.

[16] O. Adriani, G. Barbarino, G. Bazilevskaya, R. Bellotti, M. Boezio, et al., Astropart.Phys. 34, 1 (2010), 1001.3522 .

[17] S. Barwick et al. (HEAT Collaboration), Astrophys.J. 482, L191 (1997), astro-ph/9703192.

[18] M. Aguilar et al. (AMS-01 Collaboration), Phys.Lett. B646, 145 (2007), astro-ph/0703154.

[19] M. Ackermann et al. (Fermi LAT Collaboration), Phys.Rev.Lett. 108, 011103 (2012), 1109.0521.

[20] O. Adriani, G. Barbarino, G. Bazilevskaya, R. Bellotti, M. Boezio, et al., Phys.Rev.Lett. 102, 051101 (2009), 0810.4994 .

[21] O. Adriani et al. (PAMELA Collaboration), Phys.Rev.Lett. 105, 121101 (2010), 1007.0821.

[22] J. Chang, J. Adams, H. Ahn, G. Bashindzhagyan, M. Christl, et al., Nature 456, 362 (2008).

[23] F. Aharonian et al. (H.E.S.S. Collaboration), Astron.Astrophys. 508, 561 (2009), 0905.0105.

[24] J. Goodman, M. Ibe, A. Rajaraman, W. Shepherd, T. M. Tait, et al., Phys.Lett. B695, 185 (2011), 1005.1286.

[25] J. Goodman, M. Ibe, A. Rajaraman, W. Shepherd, T. M. Tait, et al., Phys.Rev. D82, 116010 (2010), 1008.1783.

[26] H. Dreiner, M. Huck, M. Kramer, D. Schmeier, and J. Tattersall, Phys.Rev. D87, 075015 (2013), 1211.2254.

[27] M. Beltran, D. Hooper, E. W. Kolb, Z. A. Krusberg, and T. M. Tait, JHEP 1009, 037 (2010), 1002.4137.

[28] P. J. Fox, R. Harnik, J. Kopp, and Y. Tsai, Phys.Rev. D85, 056011 (2012), 1109.4398.

[29] J.-M. Zheng, Z.-H. Yu, J.-W. Shao, X.-J. Bi, Z. Li, et al., Nucl.Phys. B854, 350 (2012), 1012.2022.

[30] M. R. Buckley, Phys.Rev. D84, 043510 (2011), 1104.1429 .
[31] G. Busoni, A. De Simone, E. Morgante, and A. Riotto (2013), 1307.2253.

[32] K. Griest and D. Seckel, Phys.Rev. D43, 3191 (1991).

[33] J. Edsjo and P. Gondolo, Phys.Rev. D56, 1879 (1997), hep-ph/9704361.

[34] Q.-H. Cao, C.-R. Chen, C. S. Li, and H. Zhang, JHEP 1108, 018 (2011), 0912.4511.

[35] N. Zhou, D. Berge, and D. Whiteson (2013), 1302.3619.

[36] G. Aad et al. (ATLAS Collaboration), Phys.Rev.Lett. 110, 011802 (2013), 1209.4625.

[37] N. F. Bell, J. B. Dent, A. J. Galea, T. D. Jacques, L. M. Krauss, et al., Phys.Rev. D86, 096011 (2012), 1209.0231.

[38] L. M. Carpenter, A. Nelson, C. Shimmin, T. M. Tait, and D. Whiteson (2012), 1212.3352.

[39] Y. Bai and T. M. Tait, Phys.Lett. B723, 384 (2013), 1208.4361 .

[40] G. Aad et al. (ATLAS Collaboration), Phys.Rev.Lett. 112, 041802 (2014), 1309.4017.

[41] D. Tucker-Smith and N. Weiner, Phys.Rev. D64, 043502 (2001), hep-ph/0101138.

[42] D. Tucker-Smith and N. Weiner, Phys.Rev. D72, 063509 (2005), hep-ph/0402065.

[43] Y. Bai and T. M. Tait, Phys.Lett. B710, 335 (2012), 1109.4144

[44] E. W. Kolb and M. Turner, The Early Universe (Westview Press, 1990).

[45] N. D. Christensen and C. Duhr, Comput.Phys.Commun. 180, 1614 (2009), 0806.4194.

[46] G. Belanger, F. Boudjema, A. Pukhov, and A. Semenov, Comput.Phys.Commun. 176, 367 (2007), hep$\mathrm{ph} / 0607059$.

[47] P. Ade et al. (Planck Collaboration) (2013), 1303.5062.

[48] The LEP Collaborations (2003), hep-ex/0312023.

[49] M. R. Buckley, D. Hooper, J. Kopp, and E. Neil, Phys.Rev. D83, 115013 (2011), 1103.6035.

[50] B. A. Dobrescu and F. Yu, Phys.Rev. D88, 035021 (2013), 1306.2629.

[51] J. Alwall, M. Herquet, F. Maltoni, O. Mattelaer, and T. Stelzer, JHEP 1106, 128 (2011), 1106.0522.

[52] T. Sjostrand, S. Mrenna, and P. Z. Skands, Comput.Phys.Commun. 178, 852 (2008), 0710.3820.

[53] J. de Favereau et al. (DELPHES 3), JHEP 1402, 057 (2014), 1307.6346.

[54] E. Conte, B. Fuks, and G. Serret, Comput.Phys.Commun. 184, 222 (2013), 1206.1599.

[55] The ATLAS collaboration, ATLAS-CONF-2013-082, ATLAS-COM-CONF-2013-075 (2013).

[56] The CMS Collaboration, CMS-PAS-EXO-12-048 (2013).

[57] H. An, X. Ji, and L.-T. Wang, JHEP 1207, 182 (2012), 1202.2894

[58] H. An, R. Huo, and L.-T. Wang, Phys.Dark Univ. 2, 50 (2013), 1212.2221.

[59] The ATLAS collaboration, ATLAS-CONF-2013-036, ATLAS-COM-CONF-2013-041 (2013). 